\section{Nierenkarzinom verträglicher behandeln}

\section{In der Erstlinie gehört beim metastasierten Nierenzellkarzinom (mRCC) Sunitinib zum Standard, jedoch zwingen seine Nebenwirkungen nicht selten zur Dosisreduktion. In einer Multicenter-Studie wurde retrospektiv ein womöglich verträglicheres Therapieschema untersucht.}

unitinib wird üblicherweise in einer Dosis von $50 \mathrm{mg} / \mathrm{d}$ über 4 Wochen dosiert, gefolgt von 2 Wochen Pause (4/2-Schema). Oft sind die Nebenwirkungen so stark, dass die Dosis reduziert oder die Therapie ganz unterbrochen werden muss, was Auswirkungen auf die Prognose befürchten lässt. In der retrospektiven Studie wurden Sicherheit und Effizienz eines alternativen, des in Italien häufig angewendeten 2/1-Schemas untersucht, in dem 2-wöchige Behandlungsphasen mit 1-wöchigen Ruhephasen abwechseln. Analysiert wurden die Daten von 208 Patienten, die mit Sunitinib im
4/2-Schema starteten und dann nebenwirkungsbedingt auf das 2/1-Schema wechselten (Gruppe 4/2 $\rightarrow 2 / 1$ ), sowie von 41 Patienten, die aufgrund ungünstiger klinischer Konstellationen sofort mit dem 2/1-Schema begonnen hatten (Gruppe 2/1). Als externe Kontrollgruppe dienten 211 mRCC-Patienten, die standardmäßig das 4/2-Schema erhalten hatten.

Die Rate der Nebenwirkungen Grad $\geq 3$ sank in der Gruppe 4/2 $\rightarrow$ 2/1 nach dem Wechsel signifikant von $45,7 \%$ auf $8,2 \%$ ( $<<0,001$ ), in der Gruppe 2/1 war sie mit $26,8 \%$ ähnlich hoch wie in der externen Kontrollgruppe mit 29,4\%. Die mediane Therapiedauer in den 3 Gruppen betrug 28,2, 7,8 und 9,7 Monate, die mediane progressionsfreie Überlebenszeit 30,2, 10,4 und 9,7 Monate. Das mediane Gesamtüberleben wurde in Gruppe $4 / 2 \rightarrow 2 / 1$ nicht erreicht, betrug in der 2/1-Gruppe 23,2 und in der externen Kontrollgruppe 27,8 Monate.

Fazit: Patienten mit mRCC, die unter Sunitinib aufgrund der Verträglichkeit auf ein 2/1-Therapieschema wechseln, haben weniger Nebenwirkungen als beim 4/2-Schema. Dadurch könne, so die Interpretation der Forscher, in vielen Fällen eine Reduktion der eingesetzten Dosis vermieden werden - Einbußen in puncto Wirkung und Prognose seien nicht zu befürchten. Kathrin von Kieseritzky

Bracarda S. et al. Sunitinib administered on $2 / 1$ schedule in patients with metastatic renal cell carcinoma: the RAINBOWanalysis. Ann Oncol. 2015;26(10):2107-13.

\title{
Prostatakarzinom: Wirklich geringes Risiko
}

\section{Beim Prostatakarzinom im Frühstadium ist die aktive Überwachung eine Option. In einer prospektiven Studie wurden die langfristigen Verläufe bei Patienten verfolgt, die sich dieser Strategie unterzogen hatten.}

A n der Studie nahmen 1.298 Männer im medianen Alter von 66 Jahren mit sehr niedrigem oder niedrigem $\mathrm{Ri}$ siko teil. Bei den meisten Patienten umfasste die aktive Überwachung eine halbjährlich stattfindende PSA-Messung und digitale rektale Untersuchung sowie

eine jährliche 12- bis 14-Core-Biopsie. Das mediane Follow-up betrug 5 Jahre.

49 (4\%) Patienten verstarben während des Follow-ups, die häufigste Todesursache waren kardiovaskuläre Erkrankungen, nur zwei Männer starben an ihrem Prostatakarzinom. Die Raten

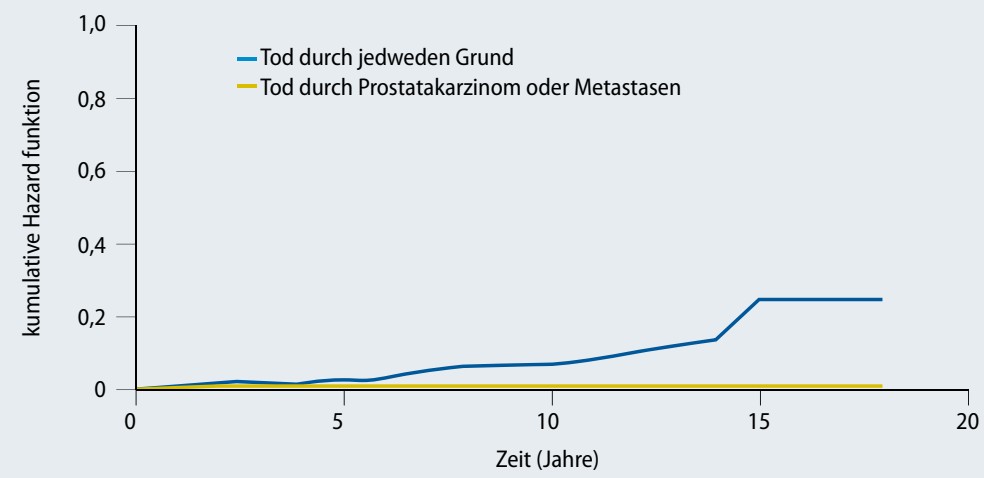

Abb. 1: Ein Tod durch das Prostatakarzinom ist bei sehr geringem Risiko selten. für Gesamtüberleben, krebsspezifisches und metastasenfreies Überleben nach 10 Jahren betrugen $93 \%$, 99,9\% und $99,4 \%$, nach 15 Jahren $69 \%, 99,9 \%$ und 99,4\%. In ein höheres Stadium musste die Erkrankung innerhalb von 10 Jahren bei $26 \%$ der Patienten reklassifiziert werden, nach 15 Jahren bei $31 \%$. Begünstigende Faktoren dafür waren ein höheres Lebensalter (Hazard Ratio 1,03 für jedes zusätzliche Jahr), eine PSA-Erhöhung und eine größere Zahl positiver Biopsiecores.

Die kumulative Inzidenz für eine kurative Intervention betrug nach 10 Jahren $50 \%$, nach 15 Jahren $57 \%$. Das mediane therapiefreie Überleben lag bei 8,5 Jahren. Mit Intervention assoziierte Faktoren waren PSA-Erhöhung und eine größere Zahl positiver Biopsiecores.

Fazit: Beim Prostatakarzinom mit günstigem Risikoprofil ist die Wahrscheinlichkeit, am Tumor zu versterben, gering, wenn sich die Patienten der aktiven Überwachung unterziehen.

Kathrin von Kieseritzky

Tosoian JJ et al. Intermediate and Longer-Term Outcomes From a Prospective Active-Surveillance Program for Favorable-Risk Prostate Cancer. J Clin Oncol. 2015;33(30):3379-85. 